\title{
FLOW PATTERNS IN HELICAL-TYPE GRAFT: BIOMEDICAL APPLICATIONS
}

\author{
E.S. BERNAD ${ }^{1}$, S.I. BERNAD ${ }^{2}$, A.F. TOTOREAN ${ }^{3}$, A.I. BOSIOC ${ }^{3} \&$ I. SARGAN ${ }^{1}$ \\ ${ }^{1}$ University of Medicine and Pharmacy 'Victor Babes' Timisoara, University Clinic 'Bega', Romania. \\ ${ }^{2}$ Romanian Academy - Timisoara Branch, Centre for Fundamental and Advanced Technical Research, Romania. \\ ${ }^{3}$ Department of MMUT, University Politehnica Timisoara, Romania.
}

\begin{abstract}
The long-term success of arterial bypass surgery is often limited by the progression of intimal hyperplasia at the anastomosis between the graft and the native artery. The experimental models were manufactured from glass tubing with a constant internal diameter of $8 \mathrm{~mm}$, fashioned into a straight configuration and helical configuration. The aim of this study was to determine the three-dimensional flow structures in the proximal anastomosis under pulsatile flow conditions and to establish the significant differences between the straight and helical graft. In the anastomosis domain, a stable region of recirculation is observed near the occluded end of the artery, which forces the flow to move into the perfused host coronary artery. A comparison between experimentally measured velocity patterns in straight and helical grafts confirms the robust nature of the secondary flows in the helical geometry. The helical configuration promotes the mixing effect of vortex motion such that the particles are mixed into the blood stream in the junction area.
\end{abstract}

Keywords: bypass graft, helical graft, particle mixing, secondary flow.

\section{INTRODUCTION}

Many factors such as compliance mismatch, effects of sutures, and the hemodynamic environment may contribute to the initiation and progression of intimal hyperplasia (IH) [1]. It has been widely reported that end-to-side vascular bypass grafts fail at the distal anastomosis [2-4].

Studies have also shown that disturbed blood flow patterns can affect the wall shear stress magnitudes and gradients, which in turn may lead to atherosclerosis and IH [5-8]. Disturbed flow patterns are initiated by a change in the geometry of the vessel through which the blood flows $[9,10]$. On the other hand, regions with low flow, that is, stagnation or recirculation, were also identified as the site of thrombotic depositions [11, 12].

Compared with traditional planar grafts, the non-planar graft configurations improve hemodynamic environments including not only promoted flow mixing and reduced stagnation flow regions, but also increased host artery vessels injury [13-15]. Caro et al. [16] carried out animal research on small amplitude helical technology (SMAHT) conduits and numerically simulated the steady flow in a four-turn helical graft. Morbiducci et al. [17] investigated the existence of a relationship between helical structure and vascular wall indices of atherogenesis in aorto-coronary bypass models. Zheng et al. numerically simulated both the steady flows and unsteady flows in helical grafts [18, 19]. Preliminary studies [20-23] suggested that the helical-type flow appeared promising in preventing $\mathrm{IH}$ and thrombosis but increased the pressure drop compared with a conventional graft [22].

The aim of this study was to determine the three-dimensional flow structures that occur at the distal anastomosis under pulsatile flow conditions and to establish the significant differences between the straight and helical graft. 
The objectives of this study were:

- To locate the flow features in the helical graft model under pulsatile flow conditions.

- To estimate the likely physical effects of the flow on the anastomosis walls of the helical graft.

\section{METHODS}

Flow structures were determined predominantly in the $60^{\circ}$ graft model, in planes parallel and perpendicular to the symmetry plane of the anastomosis (Fig. 1).

The experimental model of the graft-to-artery anastomosis is presented in Fig. 1. The proximal portion of the host artery (see Fig. 1) was occluded $3 \mathrm{~cm}$ proximal to the heel. The inlet section was sufficiently long (10 diameters) to ensure fully developed flow entering the test section. The helical graft model and the straight model have the same cross-sectional area. The helical model have the helix pitch (2D), amplitude (0.4D) and helix turn (2 turns).

Unsteady flow in the model was obtained using a computer-controlled pump at a variety of flow rates, leading to Reynolds numbers of 400 to 1000 . Both in the experimental setup and in numerical simulations, the unsteady flow model was used (Figs 2 and 3).

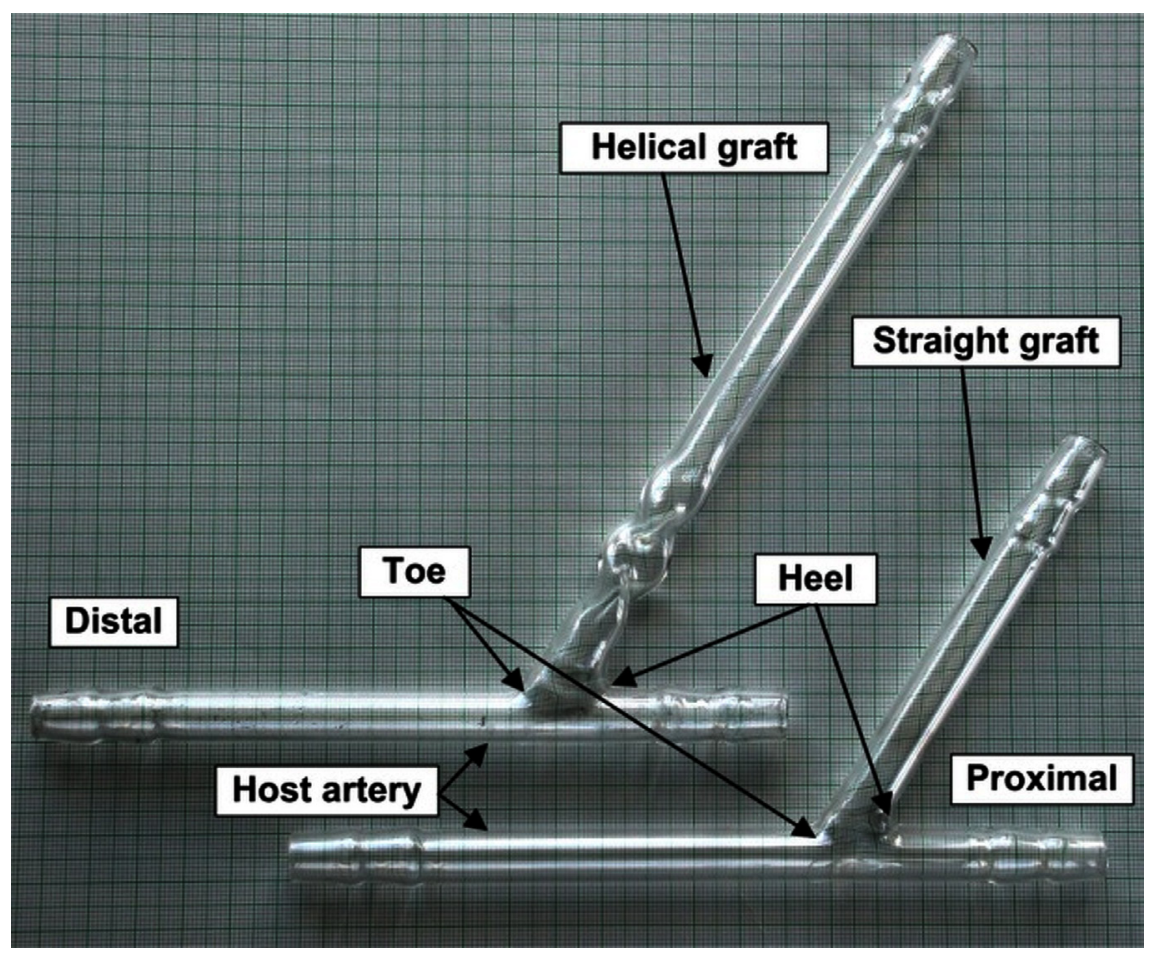

Figure 1: Experimental straight and helical bypass models manufactured from glass tubing with a constant internal diameter of $8 \mathrm{~mm}$ (for both models) used for flow visualizations. 


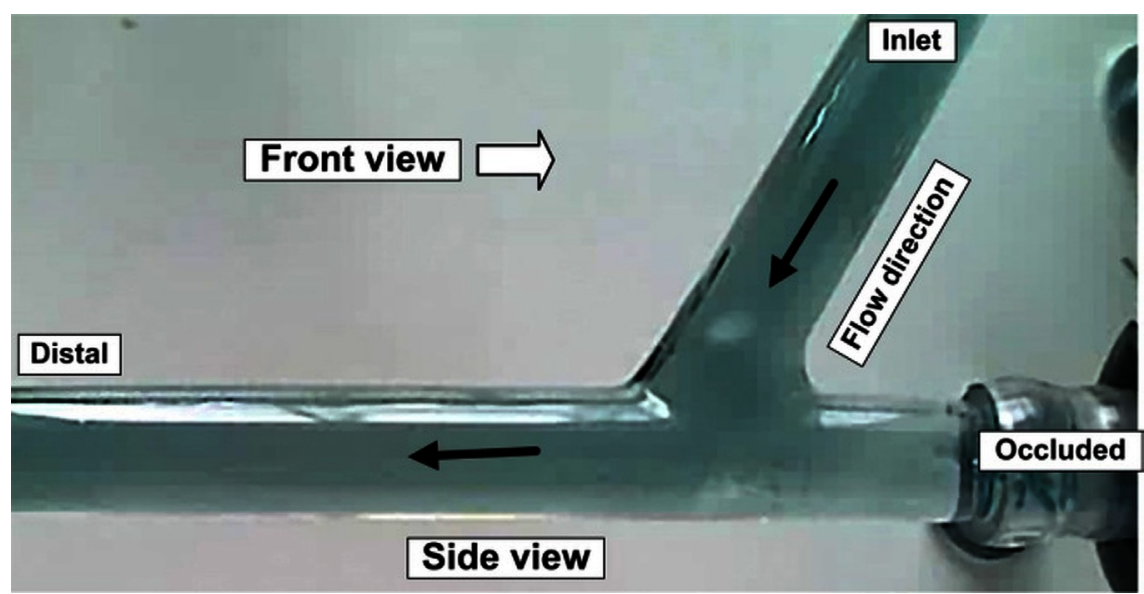

Figure 2: Straight bypass graft geometry with $60^{\circ}$ graft angle used in experimental investigations.

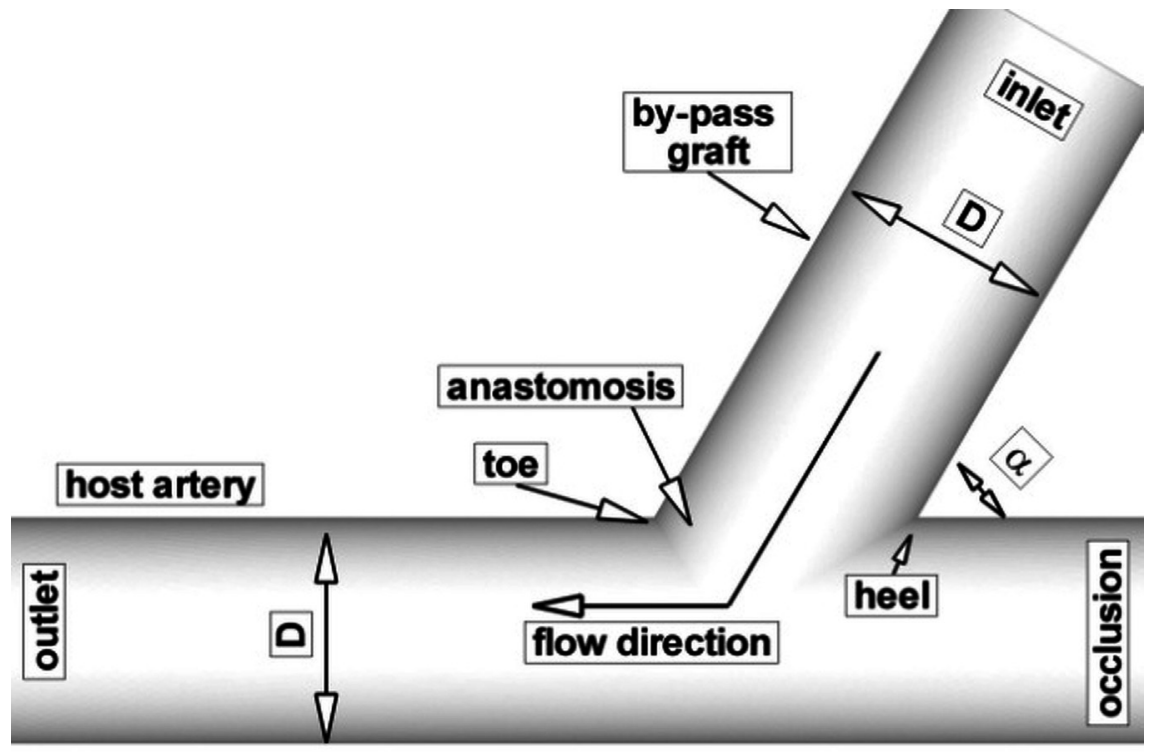

Figure 3: Straight graft model used in numerical simulations for hydrodynamic parameters investigation.

\subsection{Experimental setup}

The experimental models are fashioned using glass tubing with a constant internal diameter of $8 \mathrm{~mm}$, in a straight configuration and helical configuration with an approximately $8 \mathrm{~cm}$ straight segment proximally and $11 \mathrm{~cm}$ distally (Fig. 1).

The flow system consisted of a constant storage head tank; test section; floating ball flowmeter; collecting tank; and variable speed centrifugal pump (Fig. 4). 


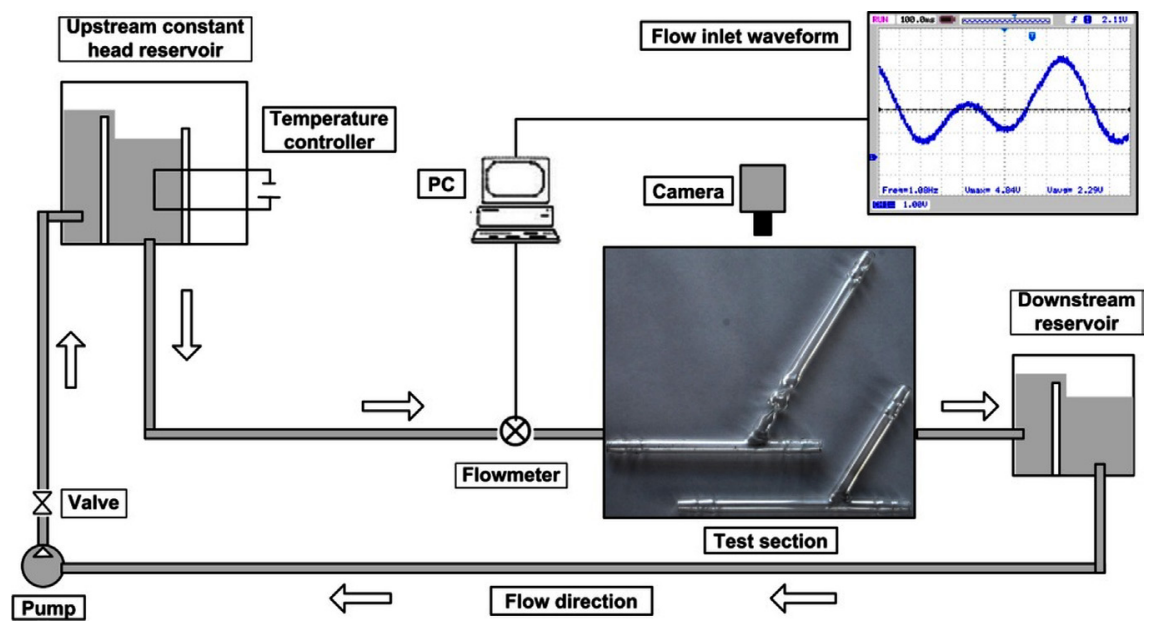

Figure 4: Experimental setup used for helical-type graft investigations. The pulsatile waveform is used in an experimental study for velocity input signal.

The mean flow rate was measured by a metric size 10 rotameter with a stainless steel float. A blood analog fluid was prepared having dynamic viscosity $(\mu)$ of $0.00408 \mathrm{~Pa}$ s and a density ( $\rho$ ) of $1050 \mathrm{~kg} / \mathrm{m}^{3}$. The flow visualization study involved the injection of a bolus of ink into blood analog fluid.

Both numerical simulations and experimental investigations have been carried out over a range of Reynolds numbers (based on the centerline temporally averaged streamwise velocity and the bypass radius) from 400 to 1000 (Fig. 5). The maximum Reynolds number during the cycle was about 1029 .

\subsection{Numerical techniques}

The authors have used simplified geometry (Fig. 3) for the by-pass graft model with graft angles of $60^{\circ}$ for analysis of the flow around the graft junction, and across the bed of the host tube. The internal diameter $\mathrm{D}$ of the graft was taken to be $8 \mathrm{~mm}$ with a graft-tube diameter ratio of 1:1 (Fig. 3). The unsteady, incompressible, three-dimensional Navier-Stokes equations were solved in Cartesian coordinates using a finite-volume-based code. Newtonian fluid behavior and rigid walls were assumed.

During the numerical simulations, the following boundary conditions must be specified, at the inlet: for the axial velocity component, a uniform inflow velocity profile and a zero transverse velocity component are used; at the outlet: the outlet pressure was defined to be $0 \mathrm{~Pa}$. The fluid is incompressible having dynamic viscosity $(\mu)$ of $0.00408 \mathrm{~Pa}$ s and a density $(\rho)$ of $1050 \mathrm{~kg} / \mathrm{m}^{3}$. The bypass walls are considered rigid and impermeable.

The $\mathrm{k}-\omega$ turbulent model used in the present simulation are capable of predicting low Reynolds number transitional flows inside the stenosed coronary artery. For blood flow numerical simulation, commercial CFD FLUENT 6.3 package was used [24]. The TECPLOT (Tecplot, Inc., Bellevue, WA, USA) software (Version 10.0) was used for analysis and quantification of the flow patterns. 


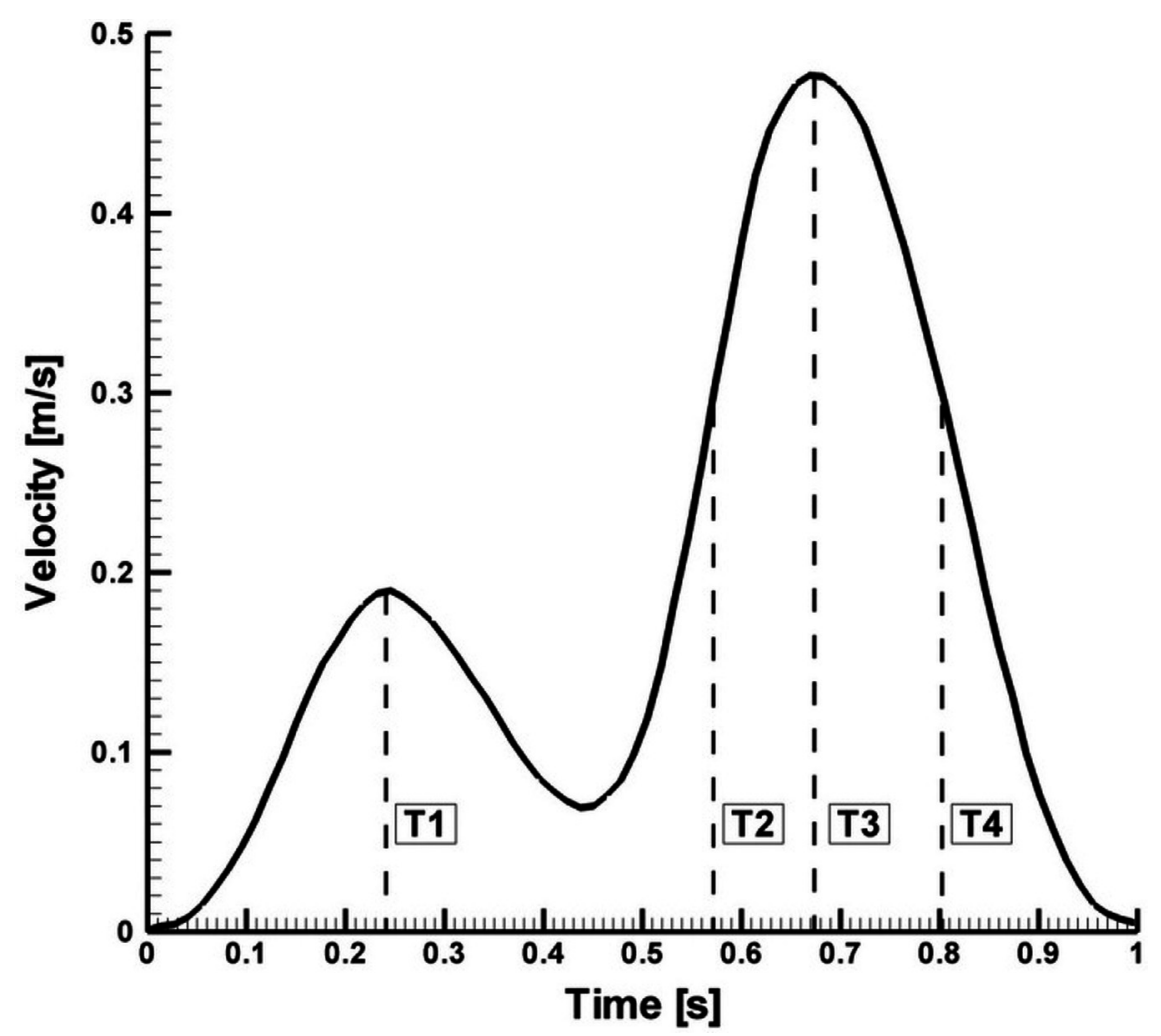

Figure 5: Pulsatile inlet flow condition. Different time steps were used both in experimental and numerical investigations.

\section{RESULTS}

The present study is motivated to understand the dynamics of flows downstream of the anastomosis section. In healthy vessels, we have a laminar flow, but in the bifurcating vessel we can have disturbed flow, leading to transition or turbulence [25].

Two model studies were undertaken to increase the understanding of the flow in a straight graft model, one involving computational fluid dynamics (CFD) and the other flow visualization. In both studies, the geometry and time-average flow were similar.

Figures 6 and 7 shows the flow structure in the $60^{\circ}$ distal anastomosis under pulsatile flow conditions corresponding to the first peak of the velocity waveform (time T1 in Fig. 5) for the experimental setup and the numerical simulations. Note the presence of two vortices in the occluded part of the bypass graft and high helical flow in the distal artery is observed in both models. The secondary vortex (V1) is the larger vortex closest to the anastomosis rotated clockwise (as viewed in the Figs 6 and 7). In the anastomosis domain, a stable region of recirculation is observed near the occluded end of the artery. This vortex forces the flow to move into the perfused host coronary (distal) artery as indicated in Figs 6 and 7.

Just downstream of the toe, at the axial position of $\mathrm{X}=0.15 \mathrm{~m}$, the axial velocity profile was skewed toward the far wall, inducing flow separation along the near wall (Figs 6 and 7). The trace at $\mathrm{X}=0.15 \mathrm{~m}$ clearly displayed the presence of secondary flow. In this region, low inertia fluid is swirling circumferentially around the vessel towards the near wall and the. The high inertia fluid from the core area is being forced toward the far wall. 


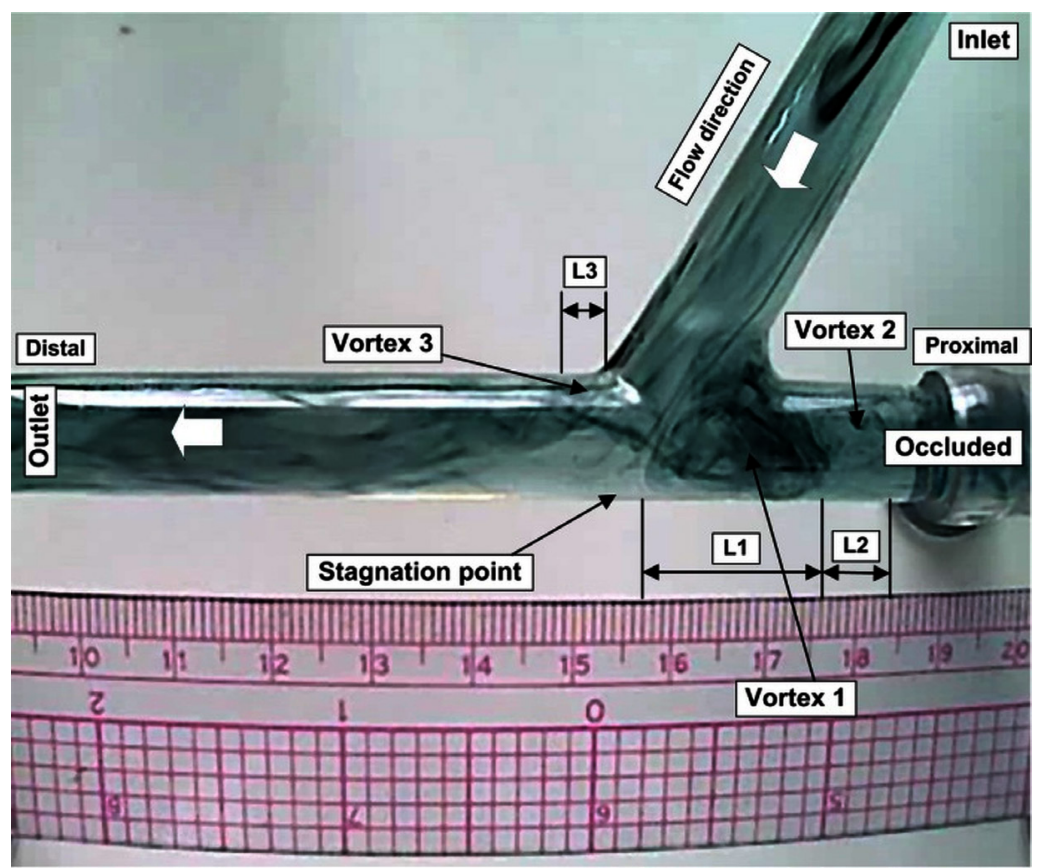

Figure 6: Flow pattern in straight bypass graft is demonstrating secondary flow. Spontaneous periodic disturbances in the distal part of the host artery.

$\alpha=60$

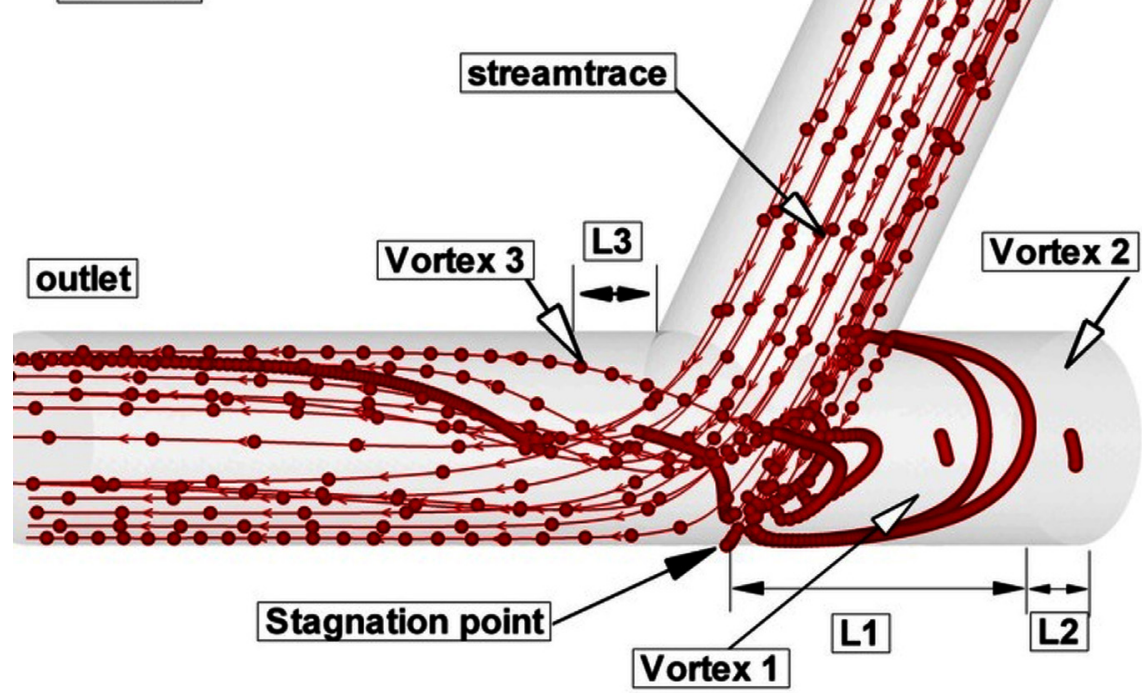

Figure 7: Flow fields in the $60^{\circ}$ graft angle geometry. A three-dimensional view of the pathline (path followed by a particle in the flow) of the high inertia fluid showing the development of the double helix (numerical results). 
Figures 6 and 7 present a good agreement between velocity patterns obtained experimentally and numerically for straight type graft. On the symmetry plane of the straight graft model, the experimental data show evidence of flow asymmetry.

Figures 8 and 9 show the flow structure in anastomotic region for time T1 and T3 associated with the $60^{\circ}$ straight graft model. In both investigated time steps, we clearly identified

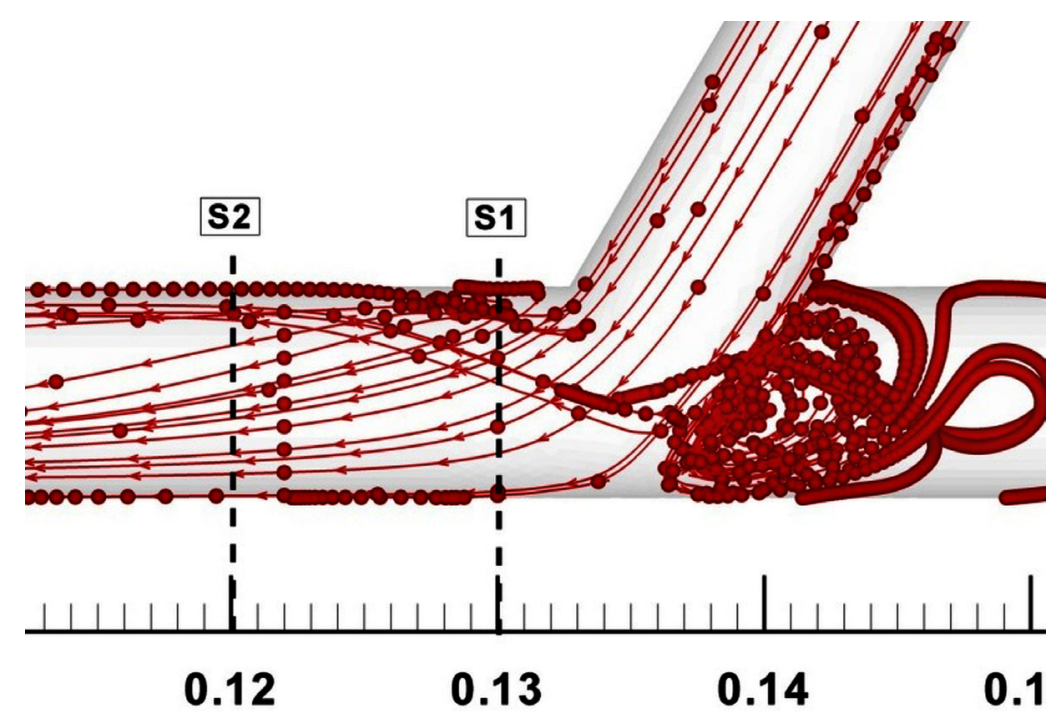

Figure 8: Particles pathline in the $60^{\circ}$ graft angle geometry corresponding to the time $\mathrm{T} 1$ (cardiac cycle presented in Fig. 5). S1 and S2 were the cross-sectional position flow field investigated.

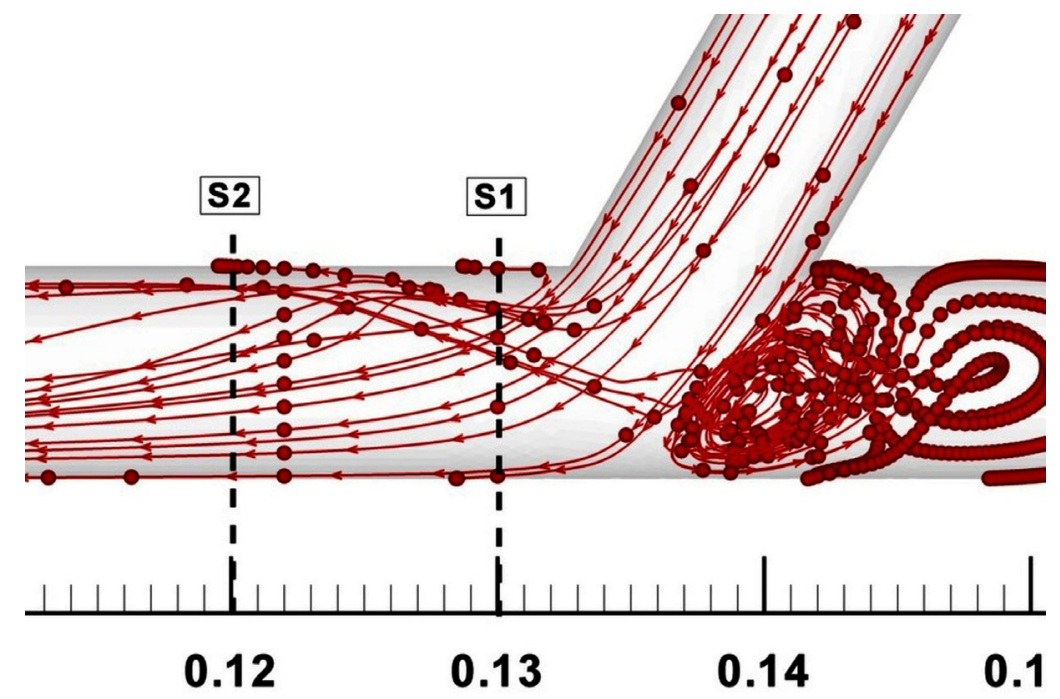

Figure 9: Particles pathline in the $60^{\circ}$ graft angle geometry corresponding to the time T3 peak flow (cardiac cycle presented in Fig. 5). S1 and S2 were the cross-sectional position flow field investigated. 
the presence of secondary flow in occluded region of the graft and a complex flow structure in the distal part of the junction.

Figures 11 and 12 show the path line for the longitudinal section through the bypass junction for anastomotic angles of $60^{\circ}$ for both bypass graft models. The highly transient character of the disturbed pulsatile flowfields became most apparent during each cycle.

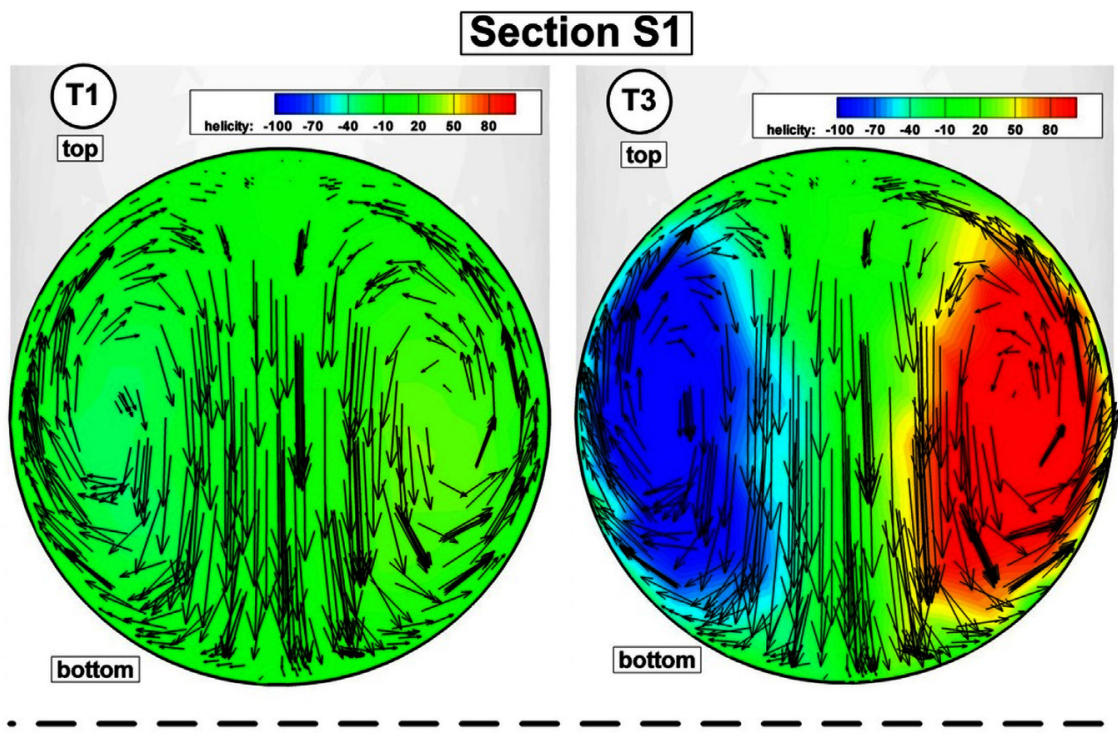

Section S2
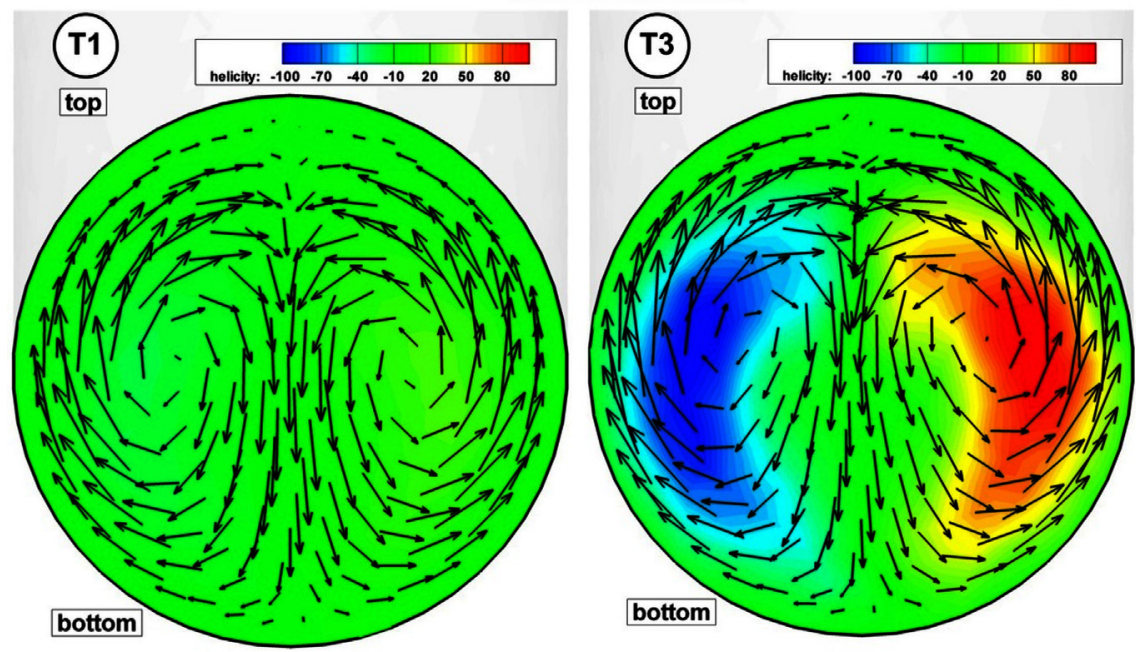

Figure 10: Secondary flow structure in investigated cross-sections S1 and S2 for time steps $\mathrm{T} 1$ and T3 (time steps are indicated in Fig. 5). Color contour of helicity field shows the complexity of the flow structure in each sections. Section positions are indicated in Figs 8 and 9). 


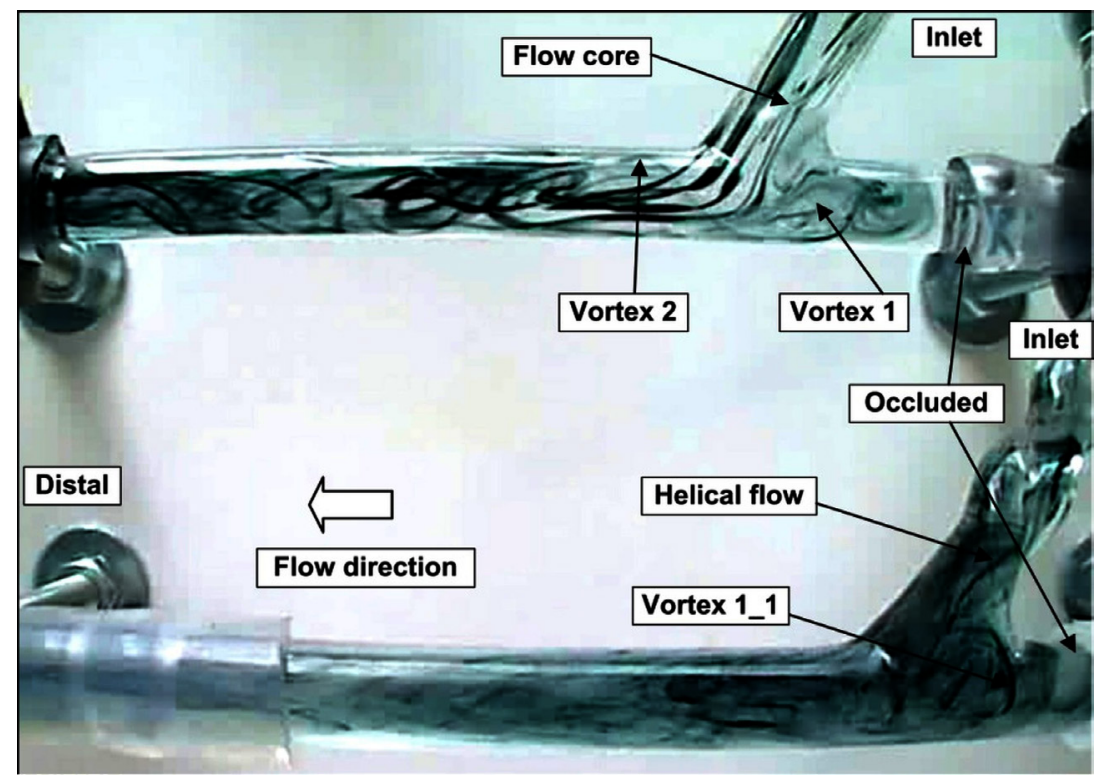

Figure 11: A comparison between flow pattern corresponding to the straight bypass graft (top) and helical-type bypass graft (bottom) (corresponding to the time T2 from Fig. 5). Flow pattern in straight graft model is demonstrating secondary flow distal to the toe. Spontaneous periodic disturbances in the distal host artery fade distally.

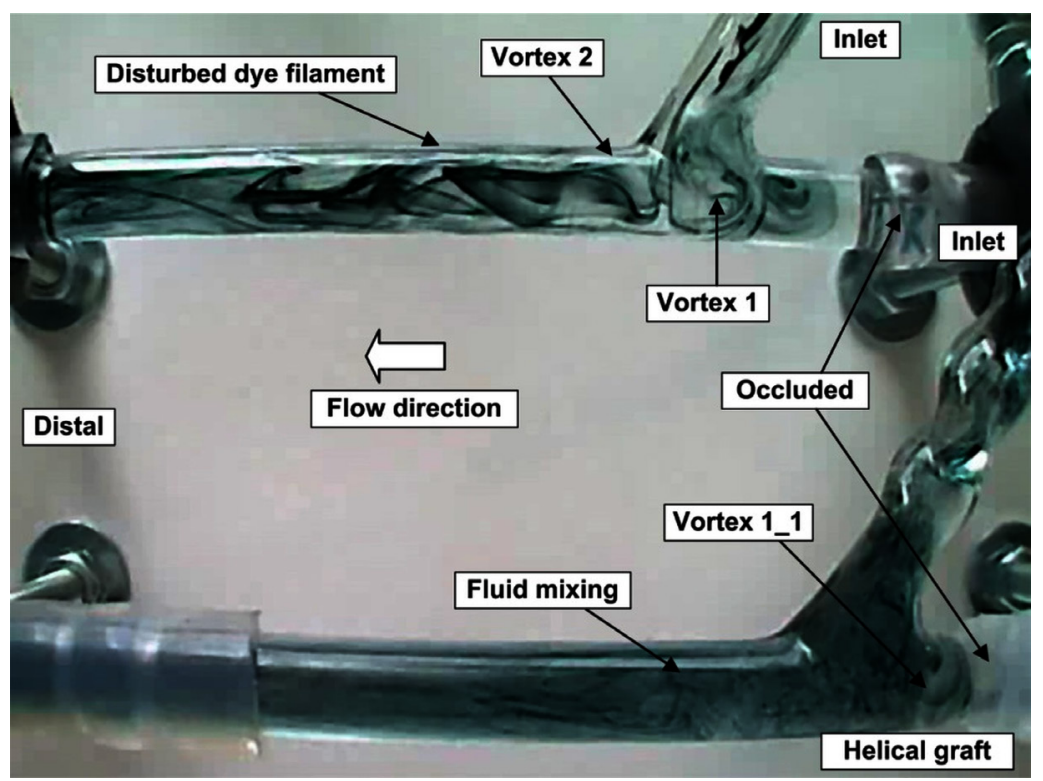

Figure 12: A comparison between flow pattern corresponding to the straight bypass graft (top) and helical-type bypass graft (bottom, corresponding to the time T4 from Fig. 5). Flow pattern in strai-ght graft model is showing some spiral reorientation of dye filaments and spontaneous periodic wave formation in the distal host artery. These periodic disturbances fade distally. 


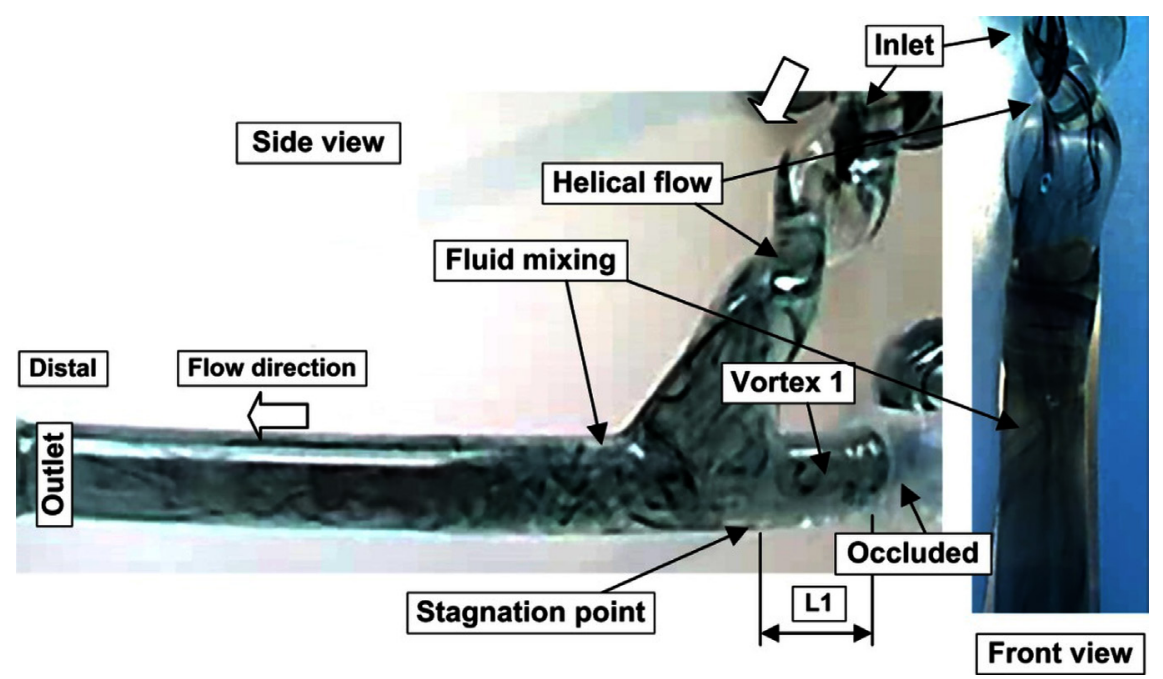

Figure 13: Flow pattern in helical graft model showing the spiral motion of dye filament inside the graft, corresponding to the time T1 (see Fig. 5).

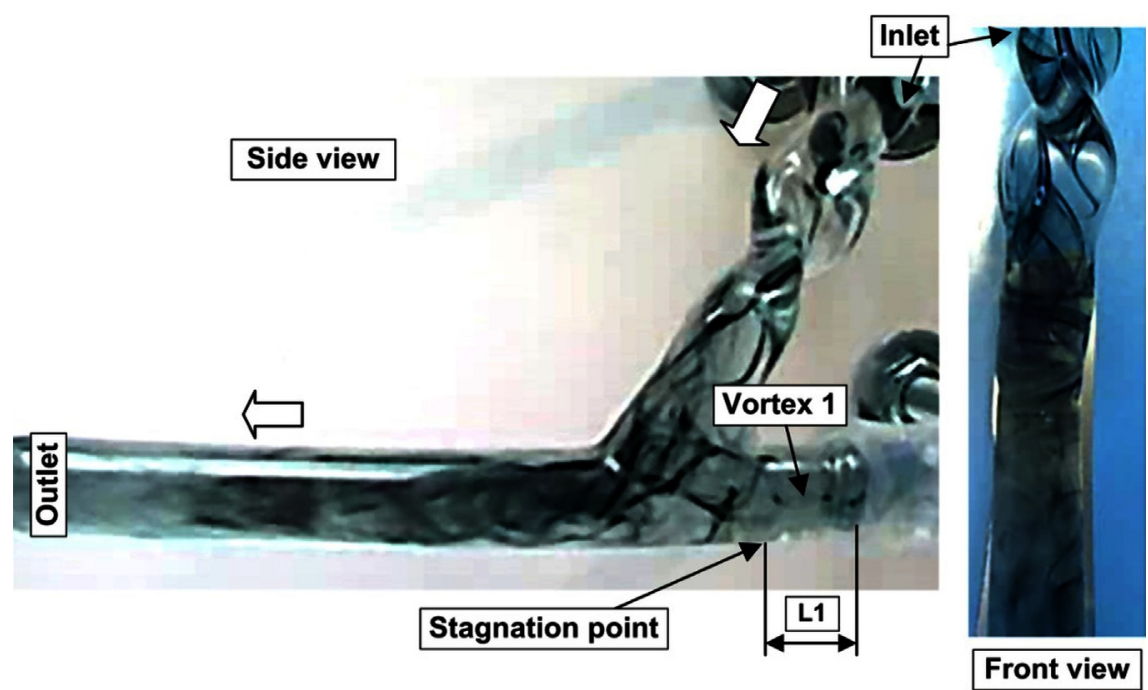

Figure 14: Flow pattern in helical graft model showing a coherent vortical structure of dye filament inside the graft, corresponding to the time T3 (see Fig. 5).

Figures 13 and 14 show the results obtained at two points in the pulsatile cycle corresponding to time T1 and T3 (Fig. 5). Figures 13 and 14 correspond to time delays of 240 and $670 \mathrm{~ms}$, respectively, relative to the start of the sinusoidal flow waveform, as shown in Fig. 5.

Figure 13 shows the flow pattern obtained during the first peak in the pulsatile cycle (time T1) and shows axial pathlines in the graft and one vortex in the occluded arterial section.

Figures 13 and 14 show that the flow structure in the graft was dominated by secondary flow patterns. 
In the occluded arterial section, the primary vortex became unstable and split into two separate smaller vortices; the most distal vortex rotated clockwise, whereas the smaller vortex, opposite the junction, rotated anti-clockwise. Secondary flow components can be seen at the toe of the graft.

In Figs 13 and 14, the proximal vortices can also be observed at the heel of the graft and illustrate the greater exchange of fluid that occurred between the occluded arterial section and the graft. Note again the significant secondary flow structures in the graft that increase the contact between blood and the graft surface.

\section{DISCUSSION}

The objective of this study was to investigate hemodynamics and wall mechanics in the distal end-to-side anastomoses to quantify the flow velocity profiles at each investigated graft models.

Arterial geometry is commonly three-dimensional [25], and the properties of inertial dominated flows in such geometries include swirling, in-plane mixing, a relatively uniform distribution of wall shear, and inhibition of flow stagnation, separation and instability [26,27]. As the flow began to decelerate, the secondary flow components became more pronounced, particularly at the toe of the graft (Fig. 9, helical type graft). The primary vortex was also larger and moved downstream into the distal artery.

In this study, mixing is only regarded as stirring flow. Fluid particles are mixed and make broad paths due to the combined effects of axial velocity and axial vorticity. In helical graft model, due to the disturbances in anastomoses vicinity and in the distal part of the host artery complete mixing of the dye occurred without the formation of parabolic curves or resumption of laminar flow (Figs 11-14).

Based on experimental data and numerical results, the following features of the velocity field can be identified:

- A core of high-momentum fluid enters the junction from the graft tube and travels towards a stagnation point on the bed of the host tube. In the neighborhood of this stagnation point, the flow splits into forward and retrograde components having large near-wall velocities (Fig. 8).

- The proximal portion of the host artery shows weak counter-rotating vortices on the symmetry plane for both models (Figs 8 and 9).

- Active secondary flows are present in the downstream section of the host tube (Figs 8-10). The magnitude of the secondary flows increases with increasing Reynolds number within the host tube (Figs 8-10).

- During the accelerating part of the second velocity peak (Fig. 2), two distinct zones of retrograde flow exist: a first region immediately distal to the toe, and a second area further downstream (Fig. 7).

In the helical graft model, the velocity enhanced near the vessel wall. We believe that the enhanced blood velocity near the vessel wall can impede the staying and adherence of platelets and leukocytes to the surface of the graft, reducing the possibility of thrombosis formation.

\section{CONCLUSIONS}

For a $60^{\circ}$ anastomotic junction, we have clearly demonstrated the complex nature of the flow pattern. To determine the relationship between hemodynamic factors and $\mathrm{IH}$, we feel that 
additional studies should be conducted to assess the influence of the angle of the anastomosis, elasticity, and other factors.

Two model studies were undertaken to increase the understanding of the flow in straight tubes, one involving CFD and the other, flow visualization. In both studies, the geometry and time-average flow were similar.

In the helical model, a mixing zone with vortex motion provides an environment for coagulant factors to be in transit without being stagnant near the surface such that the activation of the intrinsic coagulation process can be suppressed. Furthermore, the helical configuration promotes the mixing effect of vortex motion during the pulsatile cycle. The present results can provide a better understanding of the effect of geometrical configurations of helical vascular prostheses on the hemodynamic flow.

We can conclude that the geometric configuration of the distal end-to-side anastomosis can lead to flow disturbance with flow separation, which is responsible for the development of IH along the floor of the host artery. We believe that the swirling flow created in this kind of helical graft may also have an advantage of suppressing flow disturbance at the distal anastomosis.

\section{REFERENCES}

[1] Sottiurai, V.S., Yao, J.S.T., Flinn, W.R. \& Baston, R.C., Intimal hyperplasia and neointima: an ultrastructural analysis ofthrombosed grafts in humans. Surgery, 93, pp. 809-817, 1983.

[2] Ballyk, P.D., Walsh, C., Butany, J. \& Ojha, M., Compliance mismatch may promote graft-artery intimal hyperplasia by altering suture-line stresses. J. Biomech., 31, pp. 229-237, 1998.

http://dx.doi.org/10.1016/S0197-3975(97)00111-5

[3] Henry, F.S., Collins, M.W., Hughes, P.E. \& How, T.V., Numerical investigation of steady flow in proximal and distal end-to-side anastomoses. J. Biomech. Eng., 118, pp. 302-310, 1996.

http://dx.doi.org/10.1115/1.2796011

[4] Kute, S.M. \& Vorp, D.A., The effect of proximal artery flow on the hemodynamics at the distal anastomosis of a vascular bypass graft: computational study. J. Biomech. Eng., 123, pp. 277-283, 2001. http://dx.doi.org/10.1115/1.1374203

[5] Loth, F., Jones, S.A., Zarins, C.K., Giddens, D.P., Nassar, R.F., Glagov, S. \& Bassiouny, H.S., Relative contribution of wall shear stress and injury in experimental intimal thickening at eP.T.FE end-to-side arterial anastomoses. J. Biomech. Eng., 124, pp. 44-51, 2002. http://dx.doi.org/10.1115/1.1428554

[6] Hofer, M., Rappitsch, G., Perktold, K., Trubel, W. \& Schima, H., Numerical study of wall mechanics and fluid dynamics in end-to-side anastomoses and correlation to intimal hyperplasia. J. Biomech., 29(10), pp. 1297-1308, 1996. http://dx.doi.org/10.1016/0021-9290(96)00036-X

[7] Fatemi, R.S. \& Rittgers, S.E., Derivation of shear rates from near-wall LDA measurements under steady and pulsatile flow conditions. J. Biomech. Eng., 116, pp. 361-367, 1994.

http://dx.doi.org/10.1115/1.2895743 
[8] Leuprecht, A., Perktold, K., Prosi, M., Berk, T., Trubel, W. \& Schima, H., Numerical study of hemodynamics and wall mechanics in distal end-to-side anastomoses of bypass grafts. J. Biomech., 35, pp. 225-236, 2002. http://dx.doi.org/10.1016/S0021-9290(01)00194-4

[9] Quarteroni, A., Tuveri, M. \& Veneziani, A., Computational vascular dynamics: problems, models and methods. Comput. Visual. Sci., 2, pp. 163-197, 2002. http://dx.doi.org/10.1007/s007910050039

[10] Krueger, U., Zanow, J. \& Scholz, H., Computational fluid dynamics and vascular access. Artif. Organs, 26, pp. 571-575, 2002. http://dx.doi.org/10.1046/j.1525-1594.2002.07078.x

[11] Verdonck, P., The role of computational fluid dynamics for artificial organ design. Artif. Organs, 26, pp. 569-570, 2002. http://dx.doi.org/10.1046/j.1525-1594.2002.00920.x

[12] Bernad, E.S., Craina, M. \& Bernad, S.I., Blood flow simulation in patient-specific coronary bypass grafts, Proc. of the Data Management and Security, WIT Transaction on Information and Communication Technologies, eds. A. Rabasa, C.A. Brebbia \& A. Bia, WIT Press: Soauthampton, Boston, pp. 187-196, 2013.

[13] Loth, F., Fischer, P.F. \& Bassiouny, H.S., Blood flow in end-to-side anastomoses. Annu. Rev. Fluid Mech, 40, pp. 367-393, 2008.

http://dx.doi.org/10.1146/annurev.fluid.40.111406.102119

[14] Canneyt, K.V., Morbiducci, U., Eloot, S., de Sanstis, G. \& Verdock, P., A computational exploration of helical arterio-venous graft designs. J. Biomech., 46, pp. 345-353, 2013. http://dx.doi.org/10.1016/j.jbiomech.2012.10.027

[15] Bernad, S.I., Bosioc, A., Bernad, E.S. \& Craina, M.L., Comparison between experimentally measured flow patterns for straight and helical graft. Bio-Medical Materials and Engineering, 24, pp. 853-860, 2014.

[16] Caro, C.G., Nick, J.C. \& Nick, W., Preliminary comparative study of small amplitude helical and conventional ePTFE arteriovenous shunts in pigs. J. R. Soc. Interface, 2, pp. 261-266, 2005.

http://dx.doi.org/10.1098/rsif.2005.0044

[17] Morbiducci, U., Ponzini, R., Grigionid M. \& Redael, A., Helical flow as fluid dynamic signature for atherogenesis risk in aortocoronary bypass. A numeric study. J. Biomech., 40, pp. 519-534, 2007. http://dx.doi.org/10.1016/j.jbiomech.2006.02.017

[18] Zheng, T.H., Fan, Y.B., Xiong, Y., Jiang, W.T. \& Deng, X.Y., Hemodynamic performance study on small diameter helical grafts. ASAIO J., 5513, pp. 192-199, 2009.

[19] Zheng, T., Wang, W., Jiang, W., Deng, X. \& Fan, Y., Assessing hemodynamic performances of small diameter helical grafts: transient simulation. Journal of Mechanics in Medicine and Biology, 12\1, 1250008, 2012.

[20] Coppola, G. \& Caro, C., Oxygen mass transfer in a model three-dimensional artery. J. R. Soc. Interface, 5, pp. 1067-1075, 2008. http://dx.doi.org/10.1098/rsif.2007.1338

[21] Cookson, A.N., Doorly, D.J. \& Sherwin, S.J., Mixing through stirring of steady flow in small amplitude helical tubes. Ann. Biomed. Eng., 37, pp. 710-721, 2009. http://dx.doi.org/10.1007/s10439-009-9636-y

[22] Chen, Z., Fan, Y.B., Deng, X.Y. \& Xu, Z., Swirling flow can suppress flow disturbances in endovascular stents: a numerical study. ASAIO J., 55, pp. 543-549, 2009. http://dx.doi.org/10.1097/MAT.0b013e3181b78e46 
[23] Zhan, F., Fan, Y.B. \& Deng, X.Y., Swirling flow created in a glass tube suppressed platelet adhesion to the surface of the tube: its implication in the design of small-caliber arterial grafts. Thromb. Res., 125, pp. 413-418, 2010.

http://dx.doi.org/10.1016/j.thromres.2009.02.011

[24] FLUENT 6.3 User's Guide, Ansys Fluent Incorporated, 2006.

[25] Caro, C.G., Doorly, D.J., Tarnawski, M., Scott, K.T., Long, Q. \& Dumoulin, C.L., Nonplanar curvature and branching of arteries and non-planar-type flow. Proc. R. Soc., A, 452, pp. 185-197, 1996.

http://dx.doi.org/10.1098/rspa.1996.0011

[26] Caro, C.G., Watkins, N., Doorly, D.J., Sherwin, S.J. \& Peiro, J., Influence of non-planar geometry on flow separation. J. Physiol., 513, pp. 2-2, 1998.

[27] Sherwin, S.J., Shah, O., Doorly, D.J., McLean, M., Watkins, N., Caro, C.G., Peiro, J., Tarnawski, M. \& Dumoulin, C.L., Visualisation and computational study of flow at model planar and non-planar end-to-side arterial bypass grafts. J. Physiol., 504, pp. 44-44, 1997. 